\title{
On the Regularization-Homotopy Analysis Method for Linear and Nonlinear Fredholm Integral Equations of the First Kind
}

\author{
Ibrahim Issaka ${ }^{1}$, William Obeng-Denteh ${ }^{1 *}$, Isaac Owusu Mensah ${ }^{2}$, Edward Prempeh $^{1}$ \\ and Patrick Akwasi Anamuah Mensah ${ }^{1}$ \\ ${ }^{1}$ Department of Mathematics, College of Science, Kwame Nkrumah University of Science and Technology, \\ Kumasi, Ghana. \\ ${ }^{2}$ Department of Science Education, University of Education, Winneba, Mampong-Ashanti, Ghana.
}

Authors' contributions

This work was carried out in collaboration between all authors. All authors read and approved the final manuscript.

Article Information

DOI: $10.9734 /$ ARJOM/2017/32585

Editor(s):

(1) Sheng Zhang, School of Mathematics and Physics, Bohai University, Jinzhou, China.

Reviewers:

(1) H. Khalil, University of Malakand, Pakistan.

(2) P.A. Murad, Vienna, Austria.

(3) Shreekant Pathak, S V National Institute of technology, India.

(4) Nese Isler, Mehmet Akif Ersoy University, Turkey.

Complete Peer review History: http://www.sciencedomain.org/review-history/18994

Original Research Article

Received: $3^{\text {rd }}$ March 2017

Accepted: $15^{\text {th }}$ April 2017

Published: $9^{\text {th }}$ May 2017

\begin{abstract}
Fredholm integral equations of the first kind are considered by applying regularization method and the homotopy analysis method. This kind of integral equations are considered as an ill-posed problem and for this reason needs an effective method in solving them. This method first transforms a given Fredholm integral equation of the first kind to the second kind by the regularization method and then solves the transformed equation using homotopy analysis method. Approximation of the solution will be of much concern since it is not always the case to get the solution to converge and the existence of the solution is not always guaranteed as this kind of Fredholm integral equation is not well-posed.
\end{abstract}

Keywords: Regularization method; Homotopy analysis method; ill-posed problem; Fredholm integral equation of the first kind; Linear and nonlinear.

*Corresponding author: E-mail: wobengdenteh@gmail.com; 


\section{Introduction}

There have been various kinds of numerical and analytical methods used for solving Fredholm integral equations that appear in many applications in sciences and engineering. The Adomain decomposition method which was developed by Adomain in [1] and the homotopy perturbation method [2,3] have proven to work for integral, integro-differential, differential equations, linear and nonlinear types of equations. Unlike these methods, the homotopy analysis method HAM [4,5,6,7,8,9,10,11,12,13] is an analytical approximation technique which gives a reliable solution for highly nonlinear problems and also ensures rapid convergence of the solution series. Many researchers have used the HAM for linear and nonlinear problems $[14,15,16,17,18,5,13]$. In handling ill-posed problems there is the need for an effective analytic method [19,20,21,22,23,24]. In this report, the regularization method [25,26,27] and homotopy analysis method [4] are used for the first kind of Fredholm integral equations. The regularization method converts the first kind of integral equation to the second kind [28], and the homotopy analysis method is then used to solve the resulting second kind of Fredholm integral equation. A classical Fredholm integral equation of the second kind is of the form where the unknown function to be determined appears under both inside and outside the integral signs.

$$
\mu(\mathrm{x})=\mathrm{g}(\mathrm{x})+\chi \int_{a}^{b} G(x, y) \mu(y) d y
$$

Where $\mu(\mathrm{x})$ is the function to be determined, which is an unknown function. $\mathrm{g}(\mathrm{x})$ is a free term which is known, $\lambda$ is an auxiliary parameter, $a$ and $b$ are constants, the limits of the integration and $G(x, y)$ is the kernel of the integral equation.

$$
\mathrm{g}(\mathrm{x})+\chi \int_{a}^{b} G(x, y) \mu(y) d y, \mathrm{x} \in \Omega
$$

and equation (1.1.2) is the linear Fredholm integral equation of the first kind.

$$
\mathrm{g}(\mathrm{x})+\chi \int_{a}^{b} G(x, y) N(\mu(y)) d y, \mathrm{x} \in \Omega
$$

where equation (1.1.3) is the nonlinear Fredholm integral equation of the first kind and $\mathrm{N}(\mu(\mathrm{x}))$ is a nonlinear function of $\mu(\mathrm{x})$ and $\Omega$ is a closed and bounded region. These types of integral equations are often referred to as an ill-posed problems [19,20,21,22,23,24] which may either have no solution in the desired class or has more than one solutions [28].

\section{Description of the Methods}

\subsection{The regularization method}

This method was introduced in [25,26,27] by Tikhonov and Phillips respectively. Both linear and nonlinear Fredholm integral equations of the first kind is transformed by a regularization method $[25,26,27,28,20,23,24,29,30,31,32]$ to the Fredholm integral equation of the second kind. However, $\alpha$ is introduced with the unknown function to approximate Fredholm integral equation respectively as follows

$$
\alpha \mu_{\alpha}(x)=\mathrm{g}(\mathrm{x})-\int_{a}^{b} G(x, y) \mu_{\alpha}(y) d y
$$

where

$$
\alpha \mu_{\alpha}(x)=\mathrm{g}(\mathrm{x})-\int_{a}^{b} G(x, y) N\left(\mu_{\alpha}(y)\right) d y
$$


Moreover, equation 2.1.4 and 2.1.5 can be expressed as

$$
\mu_{\alpha}(x)=\frac{1}{\alpha} \mathrm{g}(\mathrm{x})-\frac{1}{\alpha} \int_{a}^{b} G(x, y) \mu_{\alpha}(y) d y
$$

and

$$
\mu_{\alpha}(x)=\frac{1}{\alpha} \mathrm{g}(\mathrm{x})-\frac{1}{\alpha} \int_{a}^{b} G(x, y) N\left(\mu_{\alpha}(y)\right) d y
$$

respectively, however (2.1.6) and (2.1.7) are second kind of Fredholm integral equation. The solution $\mu_{\alpha}(x)$ of equation 2.1 .8 or 2.1.9 usually converge to the solution $\mu(\mathrm{x})$ of (2.1.2) and (2.1.3) as $\alpha \rightarrow 0$.

Then

$$
\mu(\mathrm{x})=\lim _{\alpha \rightarrow 0} \mu_{\alpha}(x)
$$

which may not exist or be unique, for Fredholm integral equation of the first kind is an ill-posed problem.

\subsection{The homotopy analysis method}

The homotopy analysis method [14,15,16,17,10,5,6,7,8,9,10,11,12,13] was introduced by Shijun Liao in [4]. The method has successfully been used for solving linear and nonlinear equation. The homotopy analysis method gives certain advantages over other numerical methods [1,33,34] such as the perturbation method [2] which depends on small/large physical parameters and does not quarantee convergence as the equation series become bigger for strongly nonlinear problems. The HAM unlike the perturbation method [2,3], presents an accurate, fast and easy to implement for strongly nonlinear problems.

Consider the following

$$
\mathrm{N}[\mu(\tau)]=0
$$

Where $\mathrm{N}$ is a nonlinear operator, $\tau$ is an independent variable and $\mu(\tau)$ the unknown function. In constructing the zero-order deformation of the homotopy analysis method, Liao $[4,14,15,16,17,18,11,12]$ constructs,

$$
(1-\mathrm{p}) \mathrm{L}\left[\mathrm{u}(\tau ; p)-\mu_{0}(\tau)\right]=\mathrm{pz}_{0} \mathrm{H}(\tau) \mathrm{N}[\mathrm{u}(\tau ; p)-\mu(\tau)]
$$

Where $\mathrm{z}_{0} \neq 0, \mathrm{H}(\tau) \neq 0$, an auxiliary function, $\mathrm{L}$ auxiliary linear operator, $\mathrm{p} \in[0,1]$ is an embedding parameter, an interval. And as in (2.1.10) $\mu_{0}(\tau)$ represents an initial guess and $\mu(\tau)$ represents the exact solution [35]. Clearly, when

$$
\mathrm{p}=0 \text { and } \mathrm{p}=1 \text { the } \mathrm{u}(\tau, 0)=\mu_{0}(\tau) \text { and } \mathrm{u}(\tau, 1)=\mu(\tau) \text {. }
$$

As p increases from 0 to 1 , the $\mathrm{u}(\tau, p)$ changes from the $\mu_{0}(\tau)$ which is the initial guesses to $\mu(\tau)$ the exact solution.

And here we expand $\mathrm{u}(\tau, p)$ in the Taylor series with respect to $\mathrm{p}[36$, where

$$
\mathrm{u}(\tau, p)=\mu_{0}(\tau)+\sum_{n=1}^{\infty} \quad \mu_{\mathrm{n}}(\tau) \mathrm{p}^{\mathrm{n}}
$$

And here,

$$
\mu_{\mathrm{n}}(\tau)=\left.\frac{1}{\mathrm{n} !} \frac{\delta^{n} \mathrm{u}(\tau, p)}{\delta \mathrm{p}^{\mathrm{n}}}\right|_{\mathrm{p}=0}
$$


If proper selection is done with respect to the auxiliary parameter $\mathrm{h}$, the auxiliary function $\mu(\tau)$, the initial guess $\mu_{0}(\tau)$ and the auxiliary linear operator, the solution series (2.1.12) converges at where $\mathrm{p}=1$.

And

$$
\mu(\tau)=\mu_{0}(\tau)+\sum_{n=1}^{\infty} \mu_{\mathrm{n}}(\tau)
$$

The equation 2.1.14 is obtained as equation 2.1.10 is differentiated $\mathrm{n}$ times with respect to $\mathrm{p}$ and dividing by $\mathrm{n}$ ! [4] and [33]. Then the nth-order deformation equation

$$
\mathrm{L}\left[\mu_{\mathrm{n}}(\tau)-\chi_{\mathrm{n}} \mu_{\mathrm{n}-1}(\tau)\right]=\mathrm{z}_{0} \mathrm{H}(\tau) \mathrm{R}_{\mathrm{n}}\left(\mu_{n-1}(\tau)\right)
$$

And

$$
\mu_{\mathrm{n}}(\tau)=\chi_{\mathrm{n}} \mu_{\mathrm{n}-1}(\tau)+\mathrm{z}_{0} \mathrm{~L}^{-1}\left[\mathrm{H}(\tau) \mathrm{R}_{\mathrm{n}}\left(\mu_{n-1}(\tau)\right]\right.
$$

Where

$$
\mathrm{R}_{\mathrm{n}}\left(\mu_{n-1}(\tau)=\left.\frac{1}{(n-1) !} \frac{\delta^{n-1} N[u(\tau ; p)]}{\delta p^{n-1}}\right|_{\mathrm{p}=0}\right.
$$

And finally,

$$
\mu(\tau)=\sum_{n=0}^{n} \mu_{\mathrm{n}}(\tau)
$$

\subsection{Linear Fredholm integral equation of the second kind}

$$
\mu_{\alpha}(x)=\frac{1}{\alpha} \mathrm{g}(\mathrm{x})-\frac{1}{\alpha} \int_{a}^{b} G(x, y) \mu_{\alpha}(y) d y
$$

where the nth-order deformation equation is given as follows

$$
\mu_{\mathrm{n}}(\tau)=\chi_{\mathrm{n}} \mu_{\mathrm{n}-1}(\tau)+\mathrm{z}_{0} \mathrm{~L}^{-1}\left[\mathrm{H}(\tau) \mathrm{R}_{\mathrm{n}}\left(\mu_{n-1}(\tau)\right]\right.
$$

and

$$
\mathrm{R}_{\mathrm{n}}\left(\mu_{n-1}\right)=\mu_{n-1}(\mathrm{x})-\left(1-\chi_{n}\right) \frac{1}{\alpha} \mathrm{g}(\mathrm{x})+\frac{1}{\alpha} \int_{a}^{b} G(x, y) \mu_{\alpha}(y) d y
$$

Hence the solution of the nth-order deformation equation is

$$
\mu(\tau)=\sum_{n=0}^{\infty} \mu_{\mathrm{n}}(\tau)
$$

It can also be obtained by expressing

$$
\mu(\tau)=\lim _{n \rightarrow 1} \sum_{n=0}^{\infty} \quad \mu_{\alpha}(\tau) \mathrm{p}^{\mathrm{n}}
$$

hence,

$$
\begin{aligned}
& p^{0}: \mu_{0}(\tau)=\frac{1}{\alpha} \mathrm{g}(\mathrm{x}) \\
& p^{1}: \mu_{1}(\tau)=-\frac{1}{\alpha} \int_{a}^{b} G(x, t) \mu_{0}(t) d t \\
& p^{n}: \mu_{n}(\tau)=-\frac{1}{\alpha} \int_{a}^{b} G(x, t) \mu_{n-1}(t) d t
\end{aligned}
$$


After obtaining the components of $\mu_{\mathrm{i}}(\tau), \mathrm{i} \geq 0$, and finally

$$
\mu(\tau)=\sum_{n=0}^{\infty} \mu_{\mathrm{n}}(\tau)
$$

or

$$
\mu(\tau)=\lim _{n \rightarrow 1} \sum_{n=0}^{\infty} \quad \mu_{\mathrm{n}}(\tau) \mathrm{p}^{\mathrm{n}}
$$

And the series solution converges, if the solution exist

The nonlinear Fredholm integral equation of the first kind can also be treated by the regularization method [27] and [26] in the same way as in the linear case. The nonlinear first kind of Fredholm integral equation is in the form as can be seen in (3.1.24)

$$
\mathrm{g}(\mathrm{x})=\int_{a}^{b} G(x, t) N(\mu(t)) d t
$$

By the method, the equation 3.1.24 can be converted to a linear first kind of Fredholm integral equation as

$$
\mathrm{g}(\mathrm{x})=\int_{a}^{b} G(x, t) u(t) d t
$$

where

$$
\mathrm{u}(\mathrm{x})=\mathrm{N}(\mu(\mathrm{x}))
$$

and if the nonlinear function is invertible, it can be represented in this form

$$
\mu(\mathrm{x})=N^{-1}(\mathrm{u}(\mathrm{x}))
$$

There may be some difficulties in dealing with nonlinear Fredholm integral equations of the first kind due to the fact that, the equation is an ill-posed problem [28].

\subsection{Linear Fredholm integral equation of the first kind}

The linear Fredholm integral equation of the first kind is transformed to the second kind using the regularization method. The HAM is then applied to solve the problem to get approximate solutions of the equations. The existence of the solution as well as its uniqueness is not always assured [28]. For more detail about convergence, existence, and uniqueness see the following literatures [37,38,39].

Example 3.2.1. Consider the following linear first kind of Fredholm integral equation

$$
5 \mathrm{x}=5 \int_{0}^{1} x t^{3} \mu(\mathrm{t}) \mathrm{dt}
$$

By means of the regularization method, equation 3.2.28 is transformed to

$$
\alpha \mu_{\alpha}(\mathrm{x})=-5 \mathrm{x}+5 \int_{0}^{1} x t^{3} \mu_{\alpha}(\mathrm{t}) \mathrm{dt}
$$

This is reduced to the form

$$
\mu_{\alpha}(\mathrm{x})=\frac{5 x}{\alpha}-\frac{5}{\alpha} \int_{0}^{1} x t^{3} \mu_{\alpha}(\mathrm{t}) \mathrm{dt}
$$

Then equation 3.2.30 represents the second kind of Fredholm integral equation. And the nth-order homotopy deformation is constructed as 


$$
\mu_{n}(\tau)=\chi_{n} \mu_{n-1}(\tau)+\mathrm{z}_{0} L^{-1}\left[\mathrm{H}(\tau) R_{n}\left(\mu_{n-1}(\tau)\right)\right]
$$

And

$$
R_{n}\left(\mu_{n-1}(\tau)=\mu_{n-1}(\mathrm{x})-\left(1-\chi_{n}\right) \frac{5 x}{\alpha}-\frac{5}{\alpha} \int_{0}^{1} x t^{3} \mu_{\alpha}(\mathrm{t}) \mathrm{dt}\right.
$$

Where, the initial guess is set as

$$
\begin{aligned}
& \mu_{0}(\tau)=\frac{5 x}{\alpha} \\
& \mu_{1}(\tau)=-\frac{5}{\alpha} \int_{0}^{1} x t^{3} \mu_{0}(\mathrm{t}) \mathrm{dt}=-\mathrm{z}_{0} \frac{5}{\alpha^{2}} \mathrm{x} \\
& \mu_{2}(\tau)=-\frac{5}{\alpha} \int_{0}^{1} x t^{3} \mu_{1}(\mathrm{t}) \mathrm{dt}=\mathrm{z}_{0} \frac{5}{\alpha^{3}} x \\
& \mu_{3}(\tau)=-\frac{5}{\alpha} \int_{0}^{1} x t^{3} \mu_{2}(\mathrm{t}) \mathrm{dt}=-\mathrm{z}_{0} \frac{5}{\alpha^{4}} \mathrm{x} \\
& .
\end{aligned}
$$

and so on, then

$$
=5 \mathrm{x}-\mathrm{z}_{0} \frac{5}{\alpha} \mathrm{x}+\mathrm{z}_{0} \frac{5}{\alpha^{2}} \mathrm{x}-\mathrm{z}_{0} \frac{5}{\alpha^{3}} \mathrm{x} \ldots
$$

is the approximate solution.

By summing the infinite geometric solution series.

The $\mathrm{h}$ is a convergence control parameter, choosing $\mathrm{z}_{0}=-1.1$

$$
=5 \mathrm{x}+\frac{5.5}{\alpha} \mathrm{x}-\frac{5.5}{\alpha^{2}} x+\frac{5.5}{\alpha^{3}} \mathrm{x} \ldots
$$

And

$$
=5 \mathrm{x}+\frac{5.5}{\alpha} \mathrm{x}-\frac{5.5}{\alpha^{2}} x+\frac{5.5}{\alpha^{3}} \mathrm{x}-\cdots .
$$

Where

$$
=5 \mathrm{x}+\sum_{n=1}^{\infty}-5.5 x\left(\left(\frac{-1}{\alpha}\right)^{n}\right)
$$

And the

$$
\mu_{n}(\tau)=\lim _{\alpha \rightarrow 0} \sum_{n=0}^{\infty} \quad \mu_{n}(\tau)=5 \mathrm{x}
$$

Which is the exact solution. The solution may not exist and it is possible to have more than one solution.

Example 3.2.2. Consider the following linear first kind of Fredholm integral equation

$$
\frac{1}{2} \mathrm{x}=-\int_{0}^{1} \cos x t \mu(t) d t
$$

By means of the regularization method [27], equation 3.2.36 is transformed to

$$
\alpha \mu_{\alpha}(x)=\frac{1}{2} \mathrm{x}+\int_{0}^{1} \cos x t \mu_{\alpha}(t) d t
$$

Where it is reduced to the form

$$
\mu_{\alpha}(x)=\frac{1}{2 \alpha} \mathrm{x}+\frac{1}{\alpha} \int_{0}^{1} \cos x t \mu_{\alpha}(t) d t
$$


Then (3.2.38) represents the second kind of Fredholm integral equation. And the nth-order homotopy deformation is constructed as

$$
\mu_{n}(\tau)=\chi_{n} \mu_{n-1}(\tau)+\mathrm{z}_{0} L^{-1}\left[\mathrm{H}(\tau) R_{n}\left(\mu_{n-1}(\tau)\right)\right]
$$

And

$$
R_{n}\left(\mu_{n-1}(\tau)=\mu_{n-1}(\mathrm{x})-\left(1-\chi_{n}\right) \frac{1}{2 \alpha} \mathrm{x}-\frac{1}{\alpha} \int_{0}^{1} \cos x t \mu_{\alpha}(t) d t\right.
$$

Where, the initial guess is set as

$$
\begin{aligned}
& \mu_{0}(\tau)=\frac{x}{2 \alpha} \\
& \mu_{1}(\tau)=-\frac{1}{\alpha} \int_{0}^{1} \cos x t \mu_{0}(t) d t=-\mathrm{z}_{0} \frac{1}{6 \alpha^{2}} \cos x \\
& \mu_{2}(\tau)=-\frac{1}{\alpha} \int_{0}^{1} \cos x t \mu_{1}(t) d t=\mathrm{z}_{0} \frac{1}{18 \alpha^{3}} \cos x \\
& \mu_{3}(\tau)=-\frac{1}{\alpha} \int_{0}^{1} \cos x t \mu_{2}(t) d t=-\mathrm{z}_{0} \frac{1}{54 \alpha^{4}} \cos x
\end{aligned}
$$

and so on, therefore

$$
=\frac{x}{2}-\mathrm{z}_{0} \frac{1}{6 \alpha} \cos x+\mathrm{z}_{0} \frac{1}{18 \alpha^{2}} \cos x-\mathrm{z}_{0} \frac{1}{54 \alpha^{3}} \cos x \ldots
$$

For $\mathrm{z}_{0}=-1.2$

$$
=\frac{x}{2}+\frac{1.2}{6 \alpha} \cos x-\frac{1.2}{18 \alpha^{2}} \cos x+\frac{1.2}{54 \alpha^{3}} \cos x \ldots
$$

Which is the approximate solution of (3.2.36). This is achieved by summing the infinite geometric solution series.

To obtain the exact solution of equation 3.2.36, the series solution is expressed as

$$
\mu_{\alpha}(\tau)=\lim _{\alpha \rightarrow 0} \sum_{n=0}^{\infty} \quad \mu_{n}(\tau)=\frac{x}{2}
$$

And here as indicated earlier, the solution may not exist and even if it does exist its uniqueness may not be assured [25,27].

Table 1. The exact solution and RHAM result of example 3.2.1

\begin{tabular}{lllll}
\hline $\mathbf{x}$ & Exact & RHAM, $\mathbf{z}_{\mathbf{0}}=\mathbf{- 1 . 1}$ & Other solution & Error \\
\hline 0.0 & 0.000000000 & 0.000000000 & 0.000000000 & 0.000000000 \\
0.1 & 0.500000000 & 0.785185189 & 0.240740741 & -0.285185189 \\
0.2 & 1.000000000 & 1.570370376 & 0.481481482 & -0.570370376 \\
0.3 & 1.500000000 & 2.355555555 & 0.722222222 & -0.855555555 \\
0.4 & 2.000000000 & 3.140740748 & 0.962962966 & -1.140740748 \\
0.5 & 2.500000000 & 3.925925924 & 1.203703705 & -1.425925924 \\
0.6 & 3.000000000 & 4.71111111 & 1.444444445 & -1.71111111 \\
0.7 & 3.500000000 & 5.496296298 & 1.685185185 & -1.996296298 \\
0.8 & 4.000000000 & 6.281481487 & 1.925925926 & -2.281481487 \\
0.9 & 4.500000000 & 7.066666667 & 2.166666667 & -2.566666667 \\
1.0 & 5.000000000 & 7.85181843 & 2.407407409 & -2.85181843 \\
\hline
\end{tabular}


Table 2. The exact solution and RHAM result of example 3.2.36

\begin{tabular}{lllll}
\hline $\mathbf{x}$ & Exact solution & RHAM, $\mathbf{z}_{\mathbf{0}}=\mathbf{- 1 . 2}$ & Other solution & Error \\
\hline 0.0 & 0.000000000 & 0.149794217 & -0.124828514 & -0.149794214 \\
0.1 & 0.050000000 & 0.199794009 & -0.074828341 & -0.149794009 \\
0.2 & 0.100000000 & 0.249793325 & -0.02482777 & -0.149793325 \\
0.3 & 0.150000000 & 0.299792185 & 0.025173180 & -0.149792185 \\
0.4 & 0.200000000 & 0.349746721 & 0.075211066 & -0.149746721 \\
0.5 & 0.250000000 & 0.399788534 & 0.125176222 & -0.149788534 \\
0.6 & 0.300000000 & 0.449805374 & 0.175162188 & -0.149805373 \\
0.7 & 0.350000000 & 0.499783058 & 0.225180785 & -0.149783058 \\
0.8 & 0.400000000 & 0.549779636 & 0.275183636 & -0.149779636 \\
0.9 & 0.450000000 & 0.599775757 & 0.325186869 & -0.149775757 \\
1.0 & 0.500000000 & 0.649771423 & 0.37519048 & -0.149771423 \\
\hline
\end{tabular}

\subsection{The nonlinear Fredholm integral equation}

In this section, the nonlinear Fredholm integral equation is solved by the regularization-homotopy analysis method. Let us take a look at the following examples:

Example 3.2.3. Consider the following first kind of Fredholm integral equation

$$
\frac{4}{3} e^{x}=-\int_{0}^{1} t e^{x} \varphi^{2}(t) d t
$$

To start, we set

$$
\varphi^{2}(t)=\mu(t) \text { and } \varphi(t)= \pm \sqrt{\mu(t)}
$$

By changing the (3.3.43) from nonlinear Fredholm integral equation to linear Fredholm integral equation, we have

$$
\frac{4}{3} e^{x}=-\int_{0}^{1} t e^{x} \mu(t) d t
$$

And applying the regularization method, takes (3.3.45) to

$$
\mu_{\alpha}(\mathrm{x})=\frac{4}{3 \alpha} e^{x}+\frac{1}{\alpha} \int_{0}^{1} t e^{x} \mu_{\alpha}(t) d t
$$

The (3.3.46) represents the second kind of Fredholm integral equation

$$
\mu_{n}(\tau)=\chi_{n} \mu_{n-1}(\tau)+\mathrm{z}_{0} L^{-1}\left[\mathrm{H}(\tau) R_{n}\left(\mu_{n-1}(\tau)\right)\right]
$$

And

$$
R_{n}\left(\mu_{n-1}(\tau)=\mu_{n-1}(\mathrm{x})-\left(1-\chi_{n}\right) \frac{4}{3 \alpha} e^{x}-\frac{1}{\alpha} \int_{0}^{1} t e^{x} \mu_{\alpha}(t) d t\right.
$$

Then, we find the homotopy by setting the initial guess as

$$
\begin{aligned}
& \mu_{0}(\mathrm{x})=\frac{4}{3 \alpha} e^{x} \\
& \mu_{1}(\mathrm{x})=-\frac{1}{\alpha} \int_{0}^{1} t e^{x} \mu_{0}(t) d t=-\mathrm{z}_{0} \frac{4}{3 \alpha^{2}} e^{x} \\
& \mu_{2}(\mathrm{x})=-\frac{1}{\alpha} \int_{0}^{1} t e^{x} \mu_{1}(t) d t=\mathrm{z}_{0} \frac{4}{3 \alpha^{3}} e^{x}
\end{aligned}
$$




$$
\mu_{3}(\mathrm{x})=-\frac{1}{\alpha} \int_{0}^{1} t e^{x} \mu_{2}(t) d t=-\mathrm{z}_{0} \frac{4}{3 \alpha^{4}} e^{x}
$$

And this goes on and on, then we consider the approximate solution for 3.3.43. This can be achieved by summing the infinite geometric solution series.

$$
\begin{aligned}
& \mu(\mathrm{x})=\mu_{0}(\mathrm{x})+\mu_{1}(\mathrm{x})+\mu_{2}(\mathrm{x})+\mu_{3}(\mathrm{x})+\ldots \\
& \mu(\mathrm{x})=\frac{4}{3 \alpha} e^{x}-\mathrm{z}_{0} \frac{4}{3 \alpha^{2}} e^{x}+\mathrm{z}_{0} \frac{4}{3 \alpha^{3}} e^{x}-\mathrm{z}_{0} \frac{4}{3 \alpha^{4}} e^{x}+\ldots
\end{aligned}
$$

And choosing $\mathrm{z}_{0}=-1$

$$
\begin{aligned}
& \mu(\mathrm{x})=\frac{4}{3 \alpha} e^{x}+\frac{4}{3 \alpha^{2}} e^{x}-\frac{4}{3 \alpha^{3}} e^{x}+\frac{4}{3 \alpha^{4}} e^{x}+\ldots \\
& \mu(\mathrm{x})=\sqrt{\sum_{n=0}^{\infty} \mu_{n}(\mathrm{x})}
\end{aligned}
$$

For the exact solution, $\mu(\mathrm{x})$ is expressed as,

$$
\mu(x)=\lim _{\alpha \rightarrow 0} \sqrt{\sum_{n=0}^{\infty} \mu_{n}(x)}
$$

Which gives the exact solution to 3.3.43. Getting the solution series to converge [27] could be a challenge.

Example 3.2. 4. Consider this nonlinear Fredholm integral equation

$$
7 x^{2}=\int_{0}^{1} x^{2} t \varphi^{2}(\mathrm{t}) \mathrm{dt}
$$

To begin, we set

$$
\varphi^{2}(x)=\mu(x) \text { and } \varphi(x)= \pm \sqrt{\mu(x)}
$$

By changing the equation 3.3.53, from nonlinear Fredholm integral equation to linear Fredholm integral equation, we have

$$
7 x^{2}=-\int_{0}^{1} x^{2} t \mu(\mathrm{t}) \mathrm{dt}
$$

Where the regularization method is applied, takes equation 3.3.55 to

$$
\mu_{\alpha}(\mathrm{x})=\frac{7 x^{2}}{\alpha}+\frac{1}{\alpha} \int_{0}^{1} x^{2} t \mu_{\alpha}(\mathrm{t}) \mathrm{dt}
$$

And this represents the second kind of Fredholm integral equation. Introducing the nth-order homotopy deformation of (3.3.53).

$$
\mu_{n}(\tau)=\chi_{n} \mu_{n-1}(\tau)+\mathrm{z}_{0} L^{-1}\left[\mathrm{H}(\tau) R_{n}\left(\mu_{n-1}(\tau)\right)\right]
$$

And

$$
R_{n}\left(\mu_{n-1}(\tau)=\mu_{n-1}(\mathrm{x})-\left(1-\chi_{n}\right) \frac{7 x^{2}}{\alpha}-\frac{1}{\alpha} \int_{0}^{1} x^{2} t \mu_{\alpha}(\mathrm{t}) \mathrm{dt}\right.
$$


Where the initial guess is set as

$$
\begin{aligned}
& \mu_{0}(\mathrm{x})=\frac{7 x^{2}}{\alpha} \\
& \mu_{1}(\mathrm{x})=-\frac{1}{\alpha} \int_{0}^{1} x^{2} t \mu_{0}(\mathrm{t}) \mathrm{dt}=-\mathrm{z}_{0} \frac{7 x^{2}}{4 \alpha^{2}} \\
& \mu_{2}(\mathrm{x})=-\frac{1}{\alpha} \int_{0}^{1} x^{2} t \mu_{1}(\mathrm{t}) \mathrm{dt}=\mathrm{z}_{0} \frac{7 x^{2}}{16 \alpha^{3}} \\
& \mu_{3}(\mathrm{x})=-\frac{1}{\alpha} \int_{0}^{1} x^{2} t \mu_{2}(\mathrm{t}) \mathrm{dt}=-\mathrm{z}_{0} \frac{7 x^{2}}{64 \alpha^{4}} \\
&
\end{aligned}
$$

And this goes on and on, then we consider the approximate solution for (3.3.53).

$$
\begin{aligned}
& \mu(\mathrm{x})=\mu_{0}(\mathrm{x})+\mu_{1}(\mathrm{x})+\mu_{2}(\mathrm{x})+\mu_{3}(\mathrm{x})+\ldots \\
& =\frac{7 x^{2}}{\alpha}-\mathrm{z}_{0} \frac{7 x^{2}}{4 \alpha^{2}}+\mathrm{z}_{0} \frac{7 x^{2}}{16 \alpha^{3}}-\mathrm{z}_{0} \frac{7 x^{2}}{64 \alpha^{4}}+\ldots \\
& \text { If } \mathrm{z}_{0}=-\frac{1}{7} \\
& =\frac{7 x^{2}}{\alpha}+\frac{x^{2}}{4 \alpha^{2}}-\frac{x^{2}}{16 \alpha^{3}}+\frac{x^{2}}{64 \alpha^{4}}+\ldots
\end{aligned}
$$

where

$$
\mu(\mathrm{x})=\sqrt{\sum_{n=0}^{\infty} \mu_{n}(\mathrm{x})}
$$

And for the exact solution, express $\mu(\mathrm{x})$ as,

$$
\mu(\mathrm{x})=\lim _{\alpha \rightarrow 0} \sqrt{\sum_{n=0}^{\infty} \mu_{n}(\mathrm{x})}
$$

Which is the exact solution to (3.3.53). The solution if it does exist, is possible to have more than one solution and the solution may not be unique, see [25,26,27].

Table 3. The exact solution and RHAM result of example 3.3.53

\begin{tabular}{lllll}
\hline $\mathbf{x}$ & Exact solution & $\begin{array}{l}\text { RHAM, } \boldsymbol{\alpha}=\mathbf{0 . 3}, \\
\mathbf{z}_{\mathbf{0}}=\mathbf{- 1 . 1}\end{array}$ & Other solution & Error \\
\hline 0 & 0.000000000 & 0.000000000 & 0.000000000 & 0.0000000000 \\
0.1 & 0.529150262 & 0.557608865 & 0.551250074 & -0.028458603 \\
0.2 & 1.058300524 & 1.115219404 & 1.102496184 & -0.05691888 \\
0.3 & 1.587450787 & 1.672829078 & 1.636201286 & -0.085378291 \\
0.4 & 2.116601049 & 2.230438647 & 2.204992178 & -0.113837598 \\
0.5 & 2.645751311 & 2.788048453 & 2.756240491 & -0.142297142 \\
0.6 & 3.174901573 & 3.345658152 & 3.307488538 & -0.170756579 \\
0.7 & 3.704051835 & 3.903267807 & 3.858736627 & -0.199215972 \\
0.8 & 4.233202098 & 4.460877158 & 4.4099844 & -0.22767506 \\
0.9 & 4.76235236 & 5.01847234 & 4.961232813 & -0.25611998 \\
1 & 5.291502622 & 5.576096628 & 5.512483075 & -0.284594006 \\
\hline
\end{tabular}

\section{Conclusion}

The Linear and nonlinear Fredholm integral equations of the first kind are considered. The equations are transformed from Fredholm integral equation of the first kind to second kind by the regularization method 
and then solve by homotopy analysis method (HAM). This type of integral equation is an ill-posed problem which sometimes does not have a solution and where it does, its convergence and uniqueness is not always guaranteed. In this work, the approximate solutions are found and compared with the exact solutions. The regularization - homotopy analysis method showed reliability in handling Fredholm integral equation of the first kind.

\section{Competing Interests}

Authors have declared that no competing interests exist.

\section{References}

[1] Adomain G. Solving frontier problems of physics: The decomposition method. Kluwer; 1994.

[2] Aghazadeh N, Mohammadi S. A modified homotopy perturbation method for solving linear and nonlinear integral equations. International Journal of Nonlinear Sciences. 2012;3:308-316.

[3] Mahmoudi M, Kazemi MV. Solving singular BVPs ordinary differential equations by modified homotopy perturbation method. Journal of Mathematics and Computer Science. 2013;7:138-143.

[4] Liao SJ. The homotopy analysis method and its applications in mechanics. Ph.D. Thesis, Shanghai Jiaotong University; 1992.

[5] Abbasbandy S, Parkes EJ. Solitary-wave solutions of the Degasperis Procesi equation by means of the homotopy analysis method. Int. J. Comp. Math. 2010;87:2303-2313.

[6] Liao SJ. On the homotopy multiple-variable method and its applications in the interactions of nonlinear gravity waves. Commun. Nonlinear Sci. Numer. Simulat. 2011;16:1274-1303.

[7] Nui Z, Wang C. A one-step optimal homotopy analysis method for nonlinear differential equations. Commun. Nonlinear Sci. Numer. Simulat. 2010;15:2006-2036.

[8] Wu YY, Cheung KF. Homotopy solution for nonlinear differential equations in wave propagation problems. Wave Motion. 2009;46:1-14.

[9] Li YJ, Nohara BT, Liao SJ. Series solutions of coupled Van der Pol equation by means of homotopy analysis method. J. Mathematical Physis. 2010;51:063517.

DOI: $10.1063 / 1.344770$

[10] Liao SJ. Beyond perturbation-introduction to the homotopy analysis method. Chpman \& Hall/CRC Press, Boca Raton; 2003.

[11] Liao SJ. On the homotopy analysis method for nonlinear problems. Appl. Math.Comput. 2004;147: 499-513.

[12] Liao SJ. On the relationship between the homotopy analysis method and Euler transform. Commun. Nonlinear Sci. Numer. Simulat. 2010;15:1421-1431.

[13] Liao SJ. An optimal homotopy-analysis approach for strongly nonlinear differential equations. Commun. Nonlinear Sci. Numer. Simulat. 2010;15:2003-2016.

[14] Liao SJ. Advances in the homotopy analysis method. World Scientific; 2013. 
[15] Liao SJ. Homotopy analysis method in nonlinear differential equations. Springer \& Higher Education Press, Heidelberg; 2012.

[16] Zhao YL, Lin ZL, Liao SJ. An iterative HAM approach for nonlinear boundary value problems in a semi-infinite domain. Comput. Phys. Commun. 2013;184:2136-2144.

[17] Pathak S, Singh T. Optimal homotopy analysis methods for solving the linear and nonlinear Fokkerplanck equations. British Journal of Mathematics \& Computer Science, SCIENCEDOMAIN international. 2015;7(3):209-217.

[18] Abbasbandy S, Shivanian E. Predictor homotopy analysis method and its application to some nonlinear problems. Commun. Nonlinear Sci. Numer. Simulat. 2011;16:2456-2468.

[19] Solodky SG, Semenova EV. About minimal informational efforts by solving exponentially ill-posed problems. Journal of Computational \& Applied Mathematics UDC 519.642. 2015;2:119.

[20] Landi G. A discrete L-curve for the regularization of ill-posed inverse problems. University Press, Baltimore, Third Edition; 2012.

[21] Kangro I, Kangro R, Vaarmann O. Some approximate Guass-Newton -type methods for nonlinear illposed problems. Proceedings of the Estonian Academy of Sciences. 2013;62(4):227-237.

[22] Noschese S, Reichel R. A modified TSVD method for descrete ill-posed problems. VDM60Nonlinear Evolution Equation and Linear Algebra; 2013.

[23] Ge $\mathrm{X}, \mathrm{Wu} \mathrm{J}$. A new regularized solution to Ill-posed problem in coordinate transformation. International Journal of Geosciences, Scientific Research; 2012.

[24] Bleyer IR. Novel regularization methods for ill-posed problems in Hilbert and Banach spaces. University of Helsinki; 2015.

[25] Phillips DL. A technique for the numerical solution of certain integral equations of the first kind. J. Ass. Comput. Mach. 1962;9:84-96. Available:http://dx.doi.org/10.1145/321105.321114

[26] Tikhonov AN. On the solution of incorrectly posed problem and the method of regularization. Soviet Math. 1963;4:1035-1938.

[27] Tikhonov AN. Regularization of incorrectly posed problems. Soviet Math. Dokl. 1963;4:1624-1627.

[28] Wazwaz AM. The regularization - Homotopy method for the linear and nonlinear Fredholm integral equation of the first kind. Dep. of Math., Saint Xavier University, Chicago. 2011;IL 60655, USA. DOI: $10.5899 / 2011 /$ can-00105

[29] Nguyen HT, Luu VCH. Two new regularization methods for solving sideways heat equation. Journal of Inequalities and Applications, Springeropen.com; 2015.

[30] Nair TM, Pereverzev SV. Regularized collocation method for Fredholm integral equations of the first kind, ScienceDirect, Journal of COMPLEXITY; 2006.

[31] Budd CJ, Nichols NK. Tikhonov regularization for (large) inverse problems. Department of Mathematical Sciences, University of Bath. Great Western Research; 2011.

[32] Haltmeier M, Leitao A, Scherzer O. Kaczmarz methods for regularization nonlinear Ill-posed equations I: Convergence analysis. Inverse Problems and Imagine. 2007;1-298. 
[33] Shahooth MK. Numerical solution for mixed Volterra- Fredholm integral equations of the second kind by using Bernstein polynomials method. Mathematical Theory and Modeling. 2015;5:10.

[34] Rabbani M, Jamali R. Solving nonlinear system of mixed Volterra-Fredholm integral equations by using Variational iteration method. The Journal of Mathematics and Computer Science. 2012;5(4): 280-287.

[35] Issaka I, Obeng-Denteh W, Mensah PAA, Mensah IO. Homotopy analysis method for solving Fredholm integral equations of the second kind. BOMSR. 2016;4:1.

[36] Pandey PK. Non-standard finite difference method for numerical solution of second order linear Fredholm Integro-differential equations. International Journal of Mathematical Modelling \& Computations. 2015;5(3):259-266. Summer

[37] Delves LM, Mohamed JL. Computational methods for integral equations. Cambridge University Press, Cambridge; 1985.

[38] Hairer E, Norsett SP, Wanner G. Solving ordinary differential equations I Nonstiff problems (Second revised edition). Springer-Verlag New York; 1993. USA.

[39] Hu S, Wan Z, Khavanin M. On the existence and uniqueness for nonlinear integro-differential equations. Jour Math Phy. Sci. 1987;21(2):93-103.

(c) 2017 Issaka et al.; This is an Open Access article distributed under the terms of the Creative Commons Attribution License (http://creativecommons.org/licenses/by/4.0), which permits unrestricted use, distribution, and reproduction in any medium, provided the original work is properly cited.

Peer-review history:

The peer review history for this paper can be accessed here (Please copy paste the total link in your

browser address bar)

http://sciencedomain.org/review-history/18994 